\title{
Centros Especializados de San Vicente Fundación; hospital verde con certificación LEED
}

\author{
D. F. Uribe ${ }^{1, \psi}$, A.F. Arboleda ${ }^{2}$ \\ ${ }^{1}$ Gerencia Técnica Centros Especializados, San Vicente Fundación, Rionegro, Colombia \\ ${ }^{2}$ Investigaciones HUSVF, Hospital Universitario San Vicente Fundación, Medellín, Colombia
}

Recibido 1 de julio de 2015. Aceptado 15 de agosto de 2015

\section{Specialized Centers of San Vicente Fundación; LEED-certified GREEN HOSPITAL}

\author{
Centros Especializados de San Vicente Fundação; hospital verde com certificação LEED
}

\begin{abstract}
Resumen - Con el incremento de la conciencia mundial por el medio ambiente y el desarrollo de modelos de certificación y evaluación de edificaciones denominadas verdes, que apuntan a la auto-sostenibilidad, San Vicente Fundación, con la edificación de Centros Especializados, buscó ser, además de una edificación con automatización y control del consumo de energía, la primera edificación hospitalaria Verde de Colombia. De esta manera alcanzó la certificación LEED categoría plata. Dicha certificación, a la cual los proyectos se presentan voluntariamente, consiste en una medición del desempeño de la edificación en aspectos como el impacto ambiental, el consumo de recursos y la innovación. En este articulo se describen algunos aspectos de las tecnologías implementadas para desarrollar Centros Especializados como una hospital verde y a su vez, la obtención de la certificación LEED.
\end{abstract}

Palabras Clave - Hospital Verde; ahorro de energía; construcción sostenible; consumo eficiente del agua; impacto ambiental.

Abstract - With increasing global awareness about the environment and the development of models of certification and evaluation of so-called green buildings, aimed at self-sustainability, San Vicente Fundación, with the construction of specialized centers, sought to be, along with a building automation and control with energy consumption, the first green hospital in Colombia. Thus it reached LEED silver category. This certification, where projects registered voluntarily, is a measure of the performance of the building in areas such as environmental impact, resource consumption, and innovation. This article describes some aspects of the technologies implemented to develop specialized centers as a green hospital and in turn, the LEED certification.

Keywords - Green Hospital; Energy Saving; Sustainable Construction; Efficient Water Use; Environmental Impact.

Resumo-Com o aumento da consciência global sobre o meio ambiente e o desenvolvimento de modelos de certificação e de avaliação de prédios chamados verde, apontando para a auto sustentabilidade, San Vicente Fundação, com a construção de centros especializados, procurou ser, juntamente um prédio com automatização e controle do consumo de energia, o primeiro edifício

\% Dirección para correspondencia:diegouribe@amdproyectos.com.co

DOI: http:/dx.doi.org/10.14508/rbme.2015.9.18.51-56 
verde da Colômbia. Assim, alcançou a categoria prata LEED. Esta certificação, que se apresentam os projetos forma voluntária, é uma medida do desempenho do edifício em áreas como o impacto ambiental, o consumo de recursos e inovação.

Este artigo descreve alguns aspectos das tecnologias implementadas para desenvolver centros especializados como um hospital verde e, por sua vez, a certificação LEED.

Palavras chave - Hospital verde; Economia de energia; A construção sustentável; Uso eficiente da água; Impacto ambiental.

\section{INTRODUCCIÓN}

Con el objetivo de fusionar la innovación, la prestación de servicios de salud de alta tecnología, la optimización de los recursos y la disminución en el impacto ambiental, se construyó Centros Especializados; un proyecto que tiene como consigna la promoción de la salud pública, desde un enfoque de sostenibilidad, seguridad y control en su funcionamiento.

De esta manera la consigna de edificación verde, en Centros Especializados, llevó al modelo de certificación LEED a cumplir dos funciones en una misma [1]; por un lado, a los diseñadores del hospital, como una guía de diseño para una edificación de alto rendimiento y por el otro, como una herramienta de evaluación objetiva de la sostenibilidad del proyecto, bajo la modalidad verde.

Cabe resaltar que a la evaluación LEED es voluntaria y es realizada por profesionales acreditados USBGC (U.S. Building Green Council), con conocimientos en edificaciones sustentables y familiarizados con los principios y requisitos de conformidad con dicho sistema de certificación.

Dado lo anterior, se tiene por objetivo presentar cómo, mediante características de diseño, arquitectónicas, tecnológicas y de operación, el proyecto obedece a una propuesta innovadora y de excelencia que, adicionalmente se cree que la nueva sede del Hospital San Vicente en Rionegro, con su iniciativa eficiente y sostenible, tendrá influencia, en el país e internacionalmente en el ámbito latinoamericano, como modelo arquitectónico en salud.

\section{Metodología}

En Centros Especializados se cuenta con un sistema de gestión y automatización de inmuebles a gran escala, al cual están conectados servicios básicos de la edificación como la iluminación, bloqueo de puertas, detección de incendios, dispositivos hidráulicos, eléctricos y electromecánicos, que permiten monitorizar la seguridad y el control de recursos para el funcionamiento del hospital [2].

Este denominado primer hospital verde de Colombia, aplicó para la certificación LEED (Leadership in Energy and Enviromental Design), bajo la versión 2.2 de dicho modelo de evaluación. En esta versión, se evaluó bajo la modalidad de construcción nueva, en la que se distribuyen 69 posibles créditos en 6 categorías distintas:
- $\quad$ Sostenibilidad del sitio.

- Eficiencia en el manejo del agua.

- Energía y atmósfera.

- Materiales y recursos.

- Calidad del ambiente interior.

- Innovación.

Centros Especializados de San Vicente fundación, alcanzó 35 créditos de los 69 posibles, lo que le otorga una calificación de 50 puntos y lo ubica en la categoría Plata de la certificación [3].

Se llevó a cabo un detallado estudio del lote para el proyecto. Éste cuenta con un área de $106.000 \mathrm{~m}^{2}$ y todas sus condicionantes; buscando la forma más correcta de articularlo con el programa médico funcional requerido por el hospital, condujo a la elaboración de modelos (Fig.1) y a un proceso volumétrico de construcción y acomodación de conceptos en el lote (Fig. 2, Fig. 3).

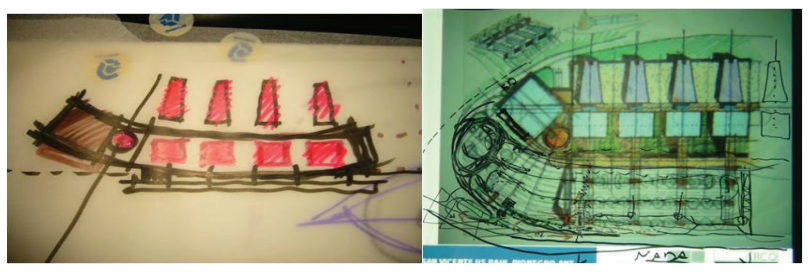

Fig. 1. Esquema de idea básica.

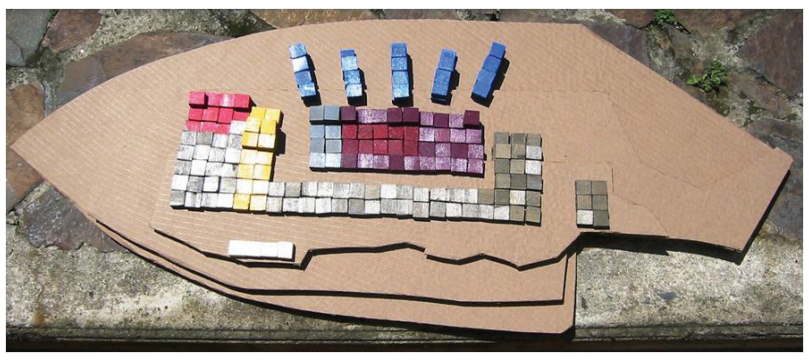

Fig. 2. Evolución a través de modelos.

Se proyectó desarrollar en una primera fase centros de excelencia para la atención especializada en las siguientes modalidades:

- Centro de Cardiopulmonar y del sistema vascular.

- Centro de Trasplantes de órganos y tejidos.

- Centro de Enfermedades digestivas.

- Centro de Urgencias.

- Hospital de Día. 


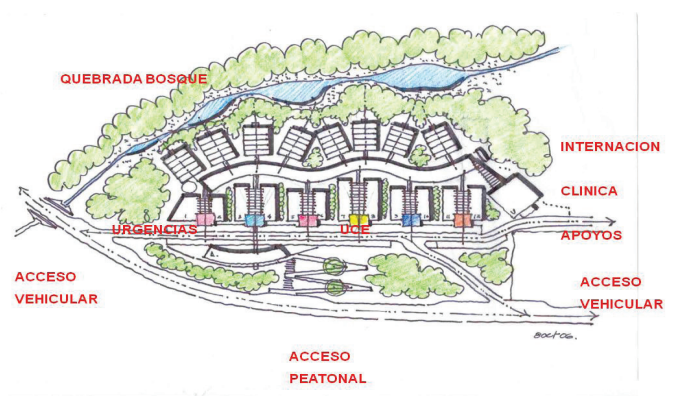

Fig. 3. Esquemas iniciales.

Se cuenta con servicios de apoyo diagnóstico y terapéutico en: Imagenología, laboratorio clínico, centro de transfusiones, unidad renal, unidad de quimioterapia, medicina física y rehabilitación, cirugía, procedimientos, unidad de cuidados intensivos, unidad de cuidados especiales e Internación de cuidado básico. Con una disponibilidad de 200 camas.

\section{RESULTADOS}

A continuación se detallan los cinco capítulos LEED y las decisiones tomadas para alcanzar los créditos y garantizar la gestión ecológica del proyecto.

\section{A. Sitios sostenibles (Puntos: 12/14)}

- Para el control de la erosión y la sedimentación se intenta controlar la erosión desde el proceso inicial de construcción.

Para reducir los impactos negativos en las fuentes de aguas y en la calidad del aire. Las medidas adoptadas para la consecución de estos objetivos han sido:

- Conservación de áreas naturales existentes $\left(15.000 \mathrm{~m}^{2}\right)$, protegiendo el bosque nativo en el retiro de la quebrada y de los nacimientos de agua, manteniéndolos como hábitat que promueven la diversidad.

- Integración del edificio en el lugar adecuado y su volumetría a la topografía del terreno (Fig. 4).

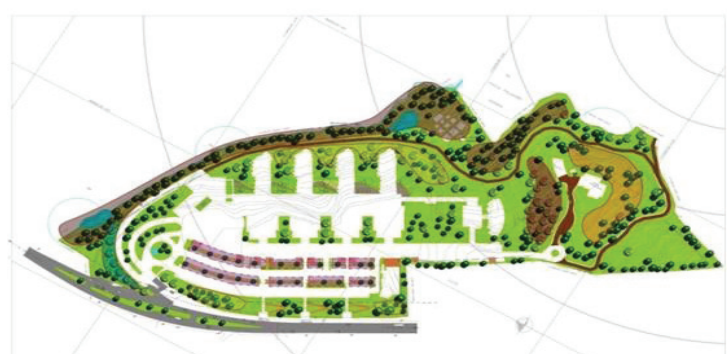

Fig. 4. Integración de la edificación con la topografía del terreno.
- El material retirado para las adecuaciones del terreno y las fundaciones está siendo utilizado para la reparación de un daño ambiental, generado por unas extracciones de áridos en la ribera del río Negro.

- Recuperación de residuos de construcción evitando generar daño ambiental con material y escombros retirados del proyecto.

- Minimización de las superficies exteriores pavimentadas en urbanización. El índice de ocupación en primer piso no alcanza el $40 \%$.

- Paisajismo exterior diseñado para reducir el efecto de islas de calor.

- Plazas de parqueo con pisos permeables que permiten la filtración del agua directamente en el suelo.

- Zonas verdes preservadas vs ocupación del lote.

- Uso de xerojardinería y empleo de especies autóctonas (Fig. 5).

- Eliminación de las cubiertas "duras" donde sea posible, sustituyéndolas por cubiertas Verdes (Healing Garden: Jardines curativos), buscando eliminar el efecto de islas calor.

- Ubicación estratégica sobre una vía con más de dos líneas de autobús público.

- Se implementarán equipamientos para parqueo de bicicletas y se fomentará el uso de la misma, pues la topografía de la zona lo permite.

- Parqueaderos preferentes para vehículos a gas o vehículos con cuatro o más ocupantes; $10 \%$ de las plazas de parqueo.

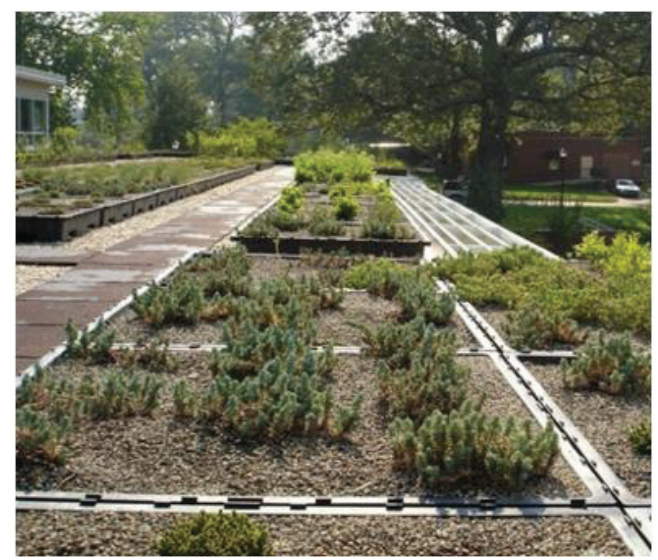

Fig. 5. Paisajismo con implementación de xerojardinería.

- Las plazas de parqueo no exceden los mínimos excedidos en la normativa local.

- Minimización de la iluminación exterior en la urbanización, reduciendo la contaminación lumínica. 


\section{B. Eficiencia en el manejo del agua (Puntos: 5/5)}

- Se propone aprovechamiento múltiple de fuentes de agua reduciendo así los consumos de las aguas municipales. Aguas de superficie, pozos profundos y aguas de lluvia y aguas recicladas.

- Eliminar el uso de agua potable para riego usando agua de lluvia y reciclada.

- Se propuso reducir los consumos interiores con la adecuación de las griferías.

- Uso de cabezales para las duchas, de alta eficiencia; grifos regulables mediante tornillo. Inodoros que pueden usarse con descarga parcial o total.

- Promoviendo el uso de aparatos sanitarios de bajo consumo y/o sin consumo (Fig. 6). Con ello pueden lograrse ahorros superiores al $20 \%$.

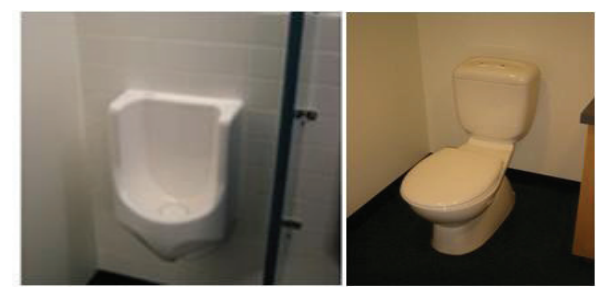

Fig. 6. Inodoros y orinales de bajo consumo.

- Esta eficacia en el uso del agua se incrementa ya que se diseñó un sistema separativo de conducciones de agua, con un uso discretizado de la misma, según se requiera; potable o no potable.

\section{Energía y atmósfera (Puntos: 7/17)}

- Utilización de energía producida con bajo impacto; a través de recursos renovables (hidroeléctricas).

- Asoleamiento e iluminación natural; como objetivo se tiene el de alcanzar unos sistemas de iluminación óptimos, al dotar al edificio de patios y vacios de luz (Fig. 7), también como lucernarios y galerías que aporten una iluminación cenital que posibilite la disminución de la utilización de las luminarias.

- Producción del $10 \%$ de la energía requerida con fuentes alternativas de energía.

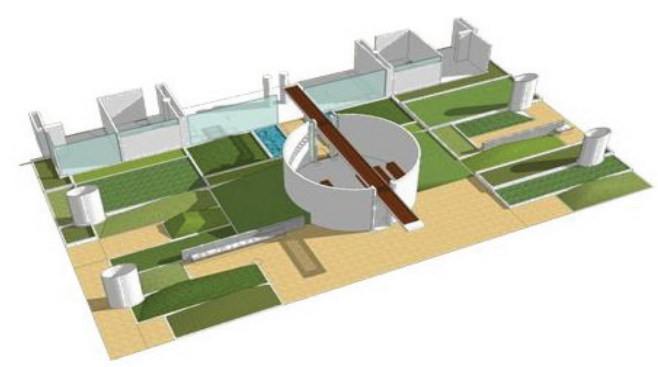

Fig. 7. Patios y vacios de luz.
- Calentamiento de agua por colectores solares (Fig. 8) y a través de recuperadoras del calor generado en la producción de aire acondicionado.

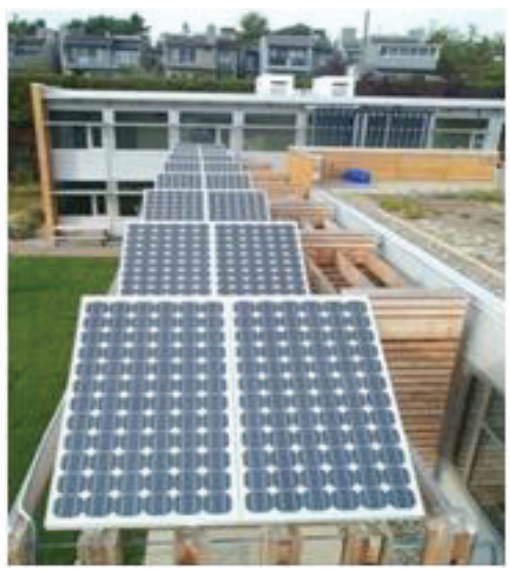

Fig. 8. Colectores solares.

- Utilización de luminarias de menor consumo y máxima regulación. En su gran mayoría, todas las luminarias del proyecto están dotadas de balastos electrónicos, los cuales además del consiguiente ahorro energético directo, permiten establecer dos mecanismos de regulación. $\mathrm{Se}$ incorporan unos detectores de presencia, que conectados al sistema domótico del proyecto, evitarán que las luminarias permanezcan encendidas cuando no se estén usando. El corte de luz se produce también cuando unas sondas luminosas situadas en el lugar más desfavorable detecten una luminosidad superior a la requerida.

- Se promueve la no utilización de equipos que contengan HCFC (hidroclorofluorocarbono) o Halon (clorofluorocarburo).

- Implementación de domótica, que ayuda a racionalizar el funcionamiento de todas y cada una de las instalaciones, permitiendo conocer de manera verídica el comportamiento de ellas y los consumos asociados a cada una. Es capital el mantenimiento y disponer de un plan para llevarlo a cabo.

- Se desarrollan procesos automatizados de control y monitoreo de redes bajo una plataforma IP con una red escalada y redundante; transporte de imágenes diagnósticas, vigilancia, acceso, comunicaciones, registros y logística.

- Se cuenta con un comisionado experto en energía que diseñe, emplee y desarrolle herramientas informáticas de simulación para optimizar la disminución en los consumos de la misma, a través del seguimiento de los reportes producidos, con los estándares ASHRAE/IESNA 90.1-1999.

\section{Materiales y recursos (Puntos: 4/13)}

- Utilización de materiales en la medida de las posibilidades de nuestro mercado cuyo origen sea sostenible; 
materiales procedentes de fuentes renovables o de procesos de reciclado y reutilización.

- Utilización de materiales de alta durabilidad y reducido mantenimiento.

- Utilización de materiales que sean susceptibles de ser reutilizados o reciclados al finalizar la vida útil del edificio [4].

- Utilizar materiales de la región o de un radio no mayor a $800 \mathrm{~km}$.

- Utilización de maderas de bosques cultivados certificados.

\section{E. Calidad del ambiente interior (Puntos 3/15)}

- Se buscó garantizar el confort; la salud y el bienestar de los ocupantes y evitar se problemas en el edificio.

- Prohibición del tabaco; se procura el edificio libre de humos y se cuenta con áreas especiales de fumadores que no contaminen el aire general.

- Monitoreo permanentemente los niveles de dióxido de carbono.

- Garantía del recambio del aire del edificio y de ser necesario, mediante ventilación mecánica.

- Reemplazo de todos los filtros del sistema de aire antes de la ocupación; luego de la pruebas del aire. Medición de las condiciones del aire.

- Utilización de materiales de baja toxicidad tanto en su fabricación como en su puesta en obra, uso, mantenimiento y posterior eliminación (respetando los limites VOC (Volatile Organic Compound)).

- Implementación de sistemas de monitoreo del aire acondicionado y de la humedad ambiente, buscando el confort térmico.

- Implementación de sistemas individuales de control de iluminación y aire acondicionado, fácilmente operables por quien ocupa un área específica, beneficiando su productividad y su salud.

- $\quad$ Proveer el máximo de iluminación natural para los espacios y visuales para los ocupantes.

\section{F. Innovación (Puntos: 5/5)}

Se obtuvieron también cinco puntos adicionales por diseños y procesos innovadores, que se listan a continuación:

1+: Plan de transporte

1+: Diseño innovador: 100\% Aguas tratadas en el sitio.

1+: Limpieza con productos biodegradables.

1+: Lámparas de bajo contenido de mercurio.

1+: Profesional con acreditación LEED.

Esto permitió consolidarse como el primer hospital verde de Colombia y uno de los primeros en Latinoamérica.

\section{Discusión}

El actual cambio cultural, donde el paciente es cada vez más consciente de sus derechos y más conocedor de su enfermedad, implica unos cambios radicales en la manera de prestar los servicios asistenciales, enfocándolos no solo en el paciente sino en la familia. Definir un espacio curativo no es fácil, pero se sabe que cuando el paciente es considerado el centro dentro de los espacios para la salud, dejando de ser un objeto de trabajo para convertirse en un sujeto de cuidados, se está en el camino correcto [5].

En el planteamiento arquitectónico y constructivo se ha dado especial relevancia a la conservación y sostenibilidad del medio ambiente y para ello se planteó el nuevo Hospital. Se tiene el enfoque en instalaciones amigables con el ambiente y eficientes en el manejo energético con estándares de diseño ecológico como el primer Hospital Verde de Colombia, para lo cual los arquitectos y constructores están siguiendo la metodología LEED ${ }^{\circ}$.

El Hospital, en su operación adoptará, en todos los procesos, estrategias de gestión ambiental que respondan a las necesidades presentes de la organización y su comunidad, para contribuir a la protección de los recursos naturales y humanos que serán necesarios en el futuro; y ello no sólo como control de la contaminación, sino como cambio cultural para pasar de un consumo excesivo de recursos a un uso de energía y elementos cada vez menor o más racional [6].

La estructuración de un complejo hospitalario a partir de unidades clínicas especializadas (Fig. 9), a las que se puede acceder desde el exterior, acogiendo el cambio en las tipologías de hospitales, dan muestra de que los espacios han evolucionado; apoyándose en la idea de que ya son cada vez más escasos los hospitales monoblock con un desmesurado tamaño, que han demostrado tener una gran dificultad en su gestión.

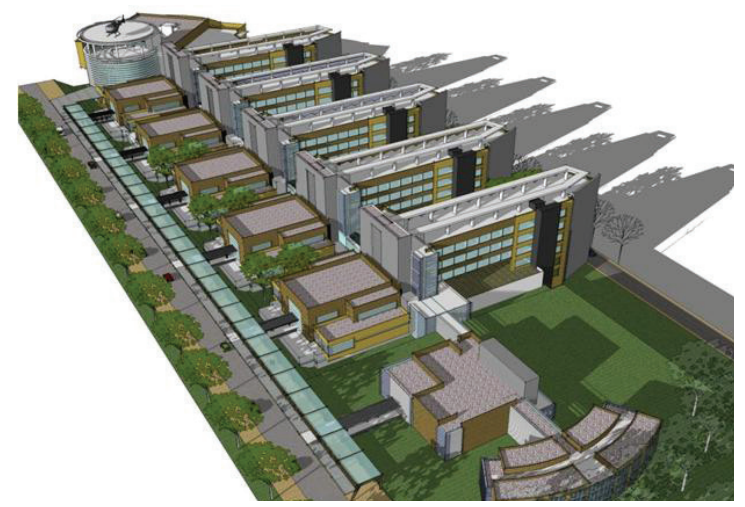

Fig. 9. Modelo virtual de distribución del espacio.

Se parte del hecho de que se tiene un diseño específico, ajustado a la concepción de los centros de excelencia con rutas de acceso independientes a los servicios y agrupados según su afinidad y complementariedad para solucionar integral- 
mente las necesidades del paciente [7]. Dichas necesidades se consolidan como la imagen visible de la nueva unidad y a partir de las cuales se orienta al paciente hacia las tecnologías nucleares y de apoyo.

\section{Conclusiones}

El sustento filosófico de la implantación del proyecto de una nueva unidad hospitalaria de alta complejidad, como polo de desarrollo, está basado en la búsqueda del liderazgo regional en la prestación de servicios asistenciales y en la generación de conocimiento e impactos positivos.

La unión del nuevo Hospital y las distintas fuerzas de la región para participar en la integración mediante la creación de redes de prestación de servicios y cadenas de abastecimiento generará la creación de nuevas fuentes de empleo y progreso para la región. Impactos favorables en el desarrollo comercial, urbanístico y turístico.

Es evidente que la nueva frontera para el diseño de Hospitales y espacios para la salud será la creación de lugares curativos que respondan a un paradigma en salud donde cuerpo y mente se unan con la tecnología moderna.

Aunque probablemente nunca habrá una fórmula fija para crear estos ambientes, está ganando reconocimiento un enfoque más holístico (del gr. holos: entero, completo) del sanar, donde adquiere relevancia la potencialidad terapéutica del ambiente construido y su entorno.

Para beneficio mutuo del ecosistema y del sector de la salud, la garantía de la protección del medio ambiente y el concepto de desarrollo sostenible hacen parte de la visión, la misión y los valores de la institución, Hospital San Vicente Fundación - Rionegro.

\section{REFERENCIAS}

[1]. Macías M., García Navarro J. Metodología y herramienta verde para la evaluación de la sostenibilidad en edificios. Informes de la construcción. 62(517), 87-100, 2010.

[2]. Congreso de la República Colombiana. Códigos colombianos de construcciones para la salud y de construcciones sismo resistentes. Resolución 4149 de 1993 y Norma sismo resistente de 1998. Bogotá.

[3]. U.S. Green Building Council. LEED certification. ScoreCard Hospital Univ. San Vicente de Paul. Consultado en mayo de 2015 en: http://www.usgbc.org/projects/ hospital-univ-san-vicente-de-paul?view=scorecard $>$.

[4]. Díaz, M. Guide of Good Environmental Practices for Building Sites: edition with OPDA Provincial Organisation for Sustainable Development . 1st ed. - Buenos Aires: Classrooms and Scaffolds, 20090 p. ISBN 978-987-24878-6-7

[5]. Green Guide for Health Care. (Agosto del 2008) Consultado en mayo de 2015 en: http://www.gghc.org/>.
[6]. Salud sin daño. Edificios saludables. Consultado en agosto de 2015 en: https://saludsindanio.org/americalatina/temas/ recursos-edificios-saludables>.

[7]. Guanteher R.. Vittori G. Sustainable healthcare architecture. Editorial John Wiley \& Sons. Edición en inglés, 2008. 429 p. ISBN 0471784044 